\title{
ELECTROSURFACE PROPERTIES OF NANOPOWDER SYSTEM BASED ON ZIRCONIA
}

\author{
${ }^{1}$ Artem SHYLO, ${ }^{1}$ Oksana GORBAN, ${ }^{1}$ Igor DANILENKO, ${ }^{1,2}$ Aleksandr DOROSHKEVICH, \\ ${ }^{3}$ Andriy LYUBCHYK, ${ }^{4}$ Anton GORBAN, ${ }^{1}$ Tetyana KONSTANTINOVA
}

${ }^{1}$ Donetsk Institute for Physics and Engineering named after O.O. Galkin, Kyiv, Ukraine, art.shylo@gmail.com

2Joint Institute for Nuclear Research, Dubna, Russian Federation, doroh@jinr.ru

3i3N/CENIMAT, Department of Materials Science, Faculty of Science and Technology, New University

of Lisbon and CEMOP/UNINOVA, Caparica, Portugal, EU, andrey.lyubchik@campus.fct.unl.pt

${ }^{4}$ Donetsk National Technical University, Pokrovsk, Donetsk region, Ukraine, anton.stroncii@gmail.com

https://doi.org/10.37904/nanocon.2019.8565

\begin{abstract}
For investigation of the influence of temperature of nanopowder calcination on adsorption properties of zirconiananoparticles, the $\mathrm{pH}$-metry method was used. As a result, structure and parameters of double electric layer surrounding zirconia nanoparticles under wetting were calculated. According presented data, titration curves of suspensions based on zirconia nanoparticles at different electrolyte concentrations intersect at values other than $\sigma_{0}=0$, which indicates the occurrence of specific sorption on the surface of zirconia nanoparticles.
\end{abstract}

Keywords: Zirconia nanoparticles, hydration, electrical double layer, pH-metry

\section{INTRODUCTION}

Zirconia is widely used in various fields of science and technology due to the unique combination of physical and chemical properties. The variety of applications is determined by the zirconia polymorphism and a wide range of surface properties. The surface properties caused by the presence of a hydration layer formed during various forms of adsorption of water molecules and other ions [1,2]. According to the Bronsted-Lewis theory, the surface of metal oxides is described as a polybasic acid or base, i.e. is a collection of different acid-base centers. The acid-base surface properties will be determined by the ratio of such centers. Studies of acid-base properties make it possible to obtain patterns of changes in acidity depending on the conditions of preparation and composition, and to obtain information on the structure of active centers.

The properties of the surface and the phase boundary of hydrated zirconia play an important role in determination their main sorption properties. The surface properties of hydrated metal oxides, such as the number of acid-base centers, surface charge, etc., change when exposed to external factors: temperature, the presence of impurities in the solution and their concentration, medium acidity, etc. Investigation of the dependence of the surface charge of hydrated metal oxides in an aqueous medium from the $\mathrm{pH}$ of solutions by establishing a zero charge point (ZP) is important for determining their sorption characteristics, studying the selectivity with respect to anions or cations and operating $\mathrm{pH}$ ranges. The point of zero charge can be determined by potentiometric titration of samples of hydrated metal oxides in solutions with different ionic strengths. The $\mathrm{pH}$ value at the intersection of several potentiometric titration curves obtained at different ionic strengths of the solutions corresponds to the $\mathrm{pH}_{\mathrm{zp}}$ value [3], at which the surface charge of the hydrated oxide is $0\left(\sigma_{0}=0\right)$ and does not depend on the concentration of the background electrolyte [4].

Investigation of the electrosurface properties of hydrated zirconia nanoparticles with different annealing temperatures by acid-base titration was the aim of this paper. The main task is to determine the point of zero charge at different concentrations of the background electrolyte. 


\section{EXPERIMENTAL}

Nanoparticles of composition $\mathrm{ZrO}_{2}-3 \% \mathrm{Y}_{2} \mathrm{O}_{3}$ with a different calcination temperature were used as an object of investigation. The technology of nanopowder obtaining by the method of co-precipitation from chloride raw material is identical to [5]. The sintering conditions of the nanopowder are $400^{\circ} \mathrm{C}$, 2hours and $700^{\circ} \mathrm{C}, 2$ hours.

The results of investigation of nanopowders for temperature of calcination of $400{ }^{\circ} \mathrm{C}$ and $700{ }^{\circ} \mathrm{C}$ by TEM method are shown on Figure 1a and Figure $1 \mathrm{~b}$ respectively. The powder is relatively well distributed spatially the particles are individualized or formed small aggregates $(60-70 \mathrm{~nm})$. The diffraction pattern corresponds to a tetragonal modification of the zirconia crystal lattice. According to the TEM data, the size of nanoparticles was 11 and $18 \mathrm{~nm}$ for nanoparticles with calcination temperature of $400^{\circ} \mathrm{C}$ and $700^{\circ} \mathrm{C}$ respectively.

Basic parameters of investigated nanopowders are presented in Table 1. As can be seen from Table 1, size of nanoparticles is increases and its specific surface area is decreases with increasing of calcinations temperature. Difference in the properties of nanoparticles causes a difference in the adsorption capacity of their surfaces.

Results of previous investigations shows a different mechanisms of sorption on surface of nanoparticles. Nanoparticles with different calcinations temperature has diferent mechanisms of water orientation - in case of particles obtained at $400{ }^{\circ} \mathrm{C}$ - mechanism of water orientation by Oxygen, at second case - by Hydrogen. This differences can caused different dielectric constant of water that surrounding of nanoparticle.

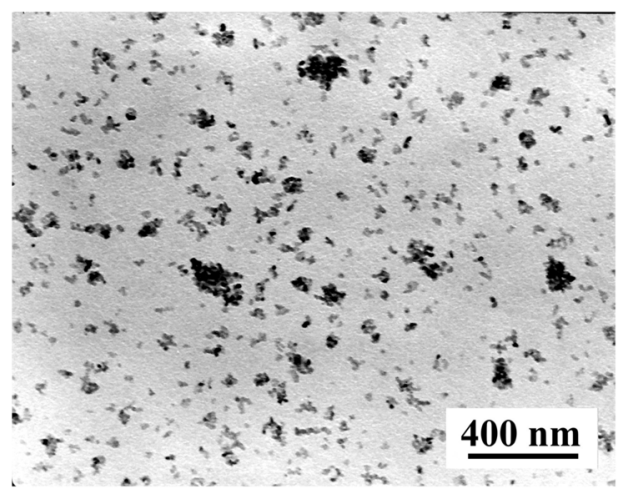

(a)

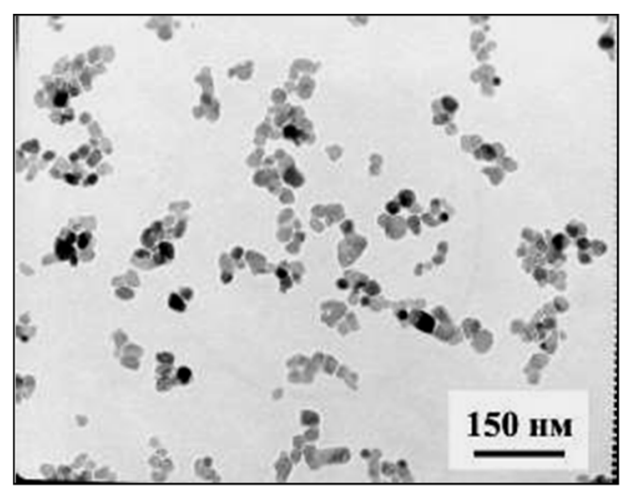

(b)

Figure 1 The TEM images of the nanopowder of composition $\mathrm{ZrO}_{2}+3 m o l \% \mathrm{Y}_{2} \mathrm{O}_{3}$, with temperature of calcination $400{ }^{\circ} \mathrm{C}(\mathrm{a})$ and $700{ }^{\circ} \mathrm{C}(\mathrm{b})$

Table 1 Zirconia nanopoarticles characteristics for different calcination temperature

\begin{tabular}{|l|l|l|}
\hline $\mathrm{ZrO}_{2}-3 \% \mathrm{Y}_{2} \mathrm{O}_{3}$ & $400{ }^{\circ} \mathrm{C}$ & $700{ }^{\circ} \mathrm{C}$ \\
\hline Phase & $96 \% \mathrm{~T}-4 \% \mathrm{M}$ & $98 \% \mathrm{~T}-2 \% \mathrm{M}$ \\
\hline $\mathrm{R}, \mathrm{nm}$ & $11(\mathrm{~T})$ & $18(\mathrm{~T})$ \\
\hline $\mathrm{S}, \mathrm{m}^{2} / \mathrm{g}$ & 109.9 & 42.2 \\
\hline
\end{tabular}

To confirm this assumption, the influence of annealing temperature of nanoparticles (i.e., different particle sizes and sorption mechanisms) on their acid properties was studied. For this, the $\mathrm{pH}$-metry method was used. The Eutech pH 700 equipment was used. To determine the $\mathrm{pH}$ of zero point $\left(\mathrm{pH}_{\mathrm{zp}}\right)$, the technique developed by Parks [6] was using. Each experiment on titration of a suspension of a sorbent in a solution corresponded to a "blank" experiment of titration of an electrolyte solution under the same conditions. The pHzp value for sorbents was found graphically as the point of intersection of the curves of the dependence of the surface charge density on the $\mathrm{pH}$ of solutions: $\sigma=\mathrm{f}(\mathrm{pH})$. The following media types were used: $\mathrm{H}_{2} \mathrm{O}, \mathrm{HCl} 0.1 \%, \mathrm{HCl} 0.01 \%$ [7]. 


\section{RESULTS AND DISCUSSION}

\subsection{The structure of a double electric layer on a surface of nanoparticles}

Upon contact of a solid with an electrolyte solution, a number of processes occur leading to charging of a surface. Near the charged surface, the ion concentrations changes: ions of opposite sign are attracted to a surface from a solution and ions of the same sign with the charge of a surface are repelled. As a result, a socalled double electric layer is formed in the solid body / electrolyte system. In case with zirconia nanoparticles, surface charging occurs due to the polarization of the surface of nanoparticles. Allocate 1) a dense part of the double layer (Stern layer) and 2) diffuse part [8]. The dense part is generally represented in the form of inner and outer parts. The inner part is formed by dehydrated ions adsorbed on a given surface. Since the ions have a finite size, there is a difference between the potential of the wall $\varphi_{0}$ and the potential $\varphi_{d}$ in that layer (the internal Helmholtz layer) in which the adsorbed dehydrated ions are located (in the Stern layer). The potential $\varphi_{d}$ is often called Stern potential. The outer part of the dense layer is formed by hydrated ions, the interaction of which with the surface is practically devoid of specificity. The so-called outer Helmholtz layer is associated with the position of these ions. Behind the outer Helmholtz layer is the diffuse part of the double layer (the Gui layer), into which sometimes the ions located in the outer plane of Helmholtz are included $[9,10]$.

In this case, the structure of the double layer determines not only thermodynamic, but also electrokinetic characteristics of the surface of colloidal systems. That is, different mechanisms of water sorption on the surface of nanoparticles with different calcination temperatures should influence the parameters of the double electrical layer surrounding the nanoparticle, which may be important for determining of the mechanism of generation and transfer of charge by zirconia nanoparticles.

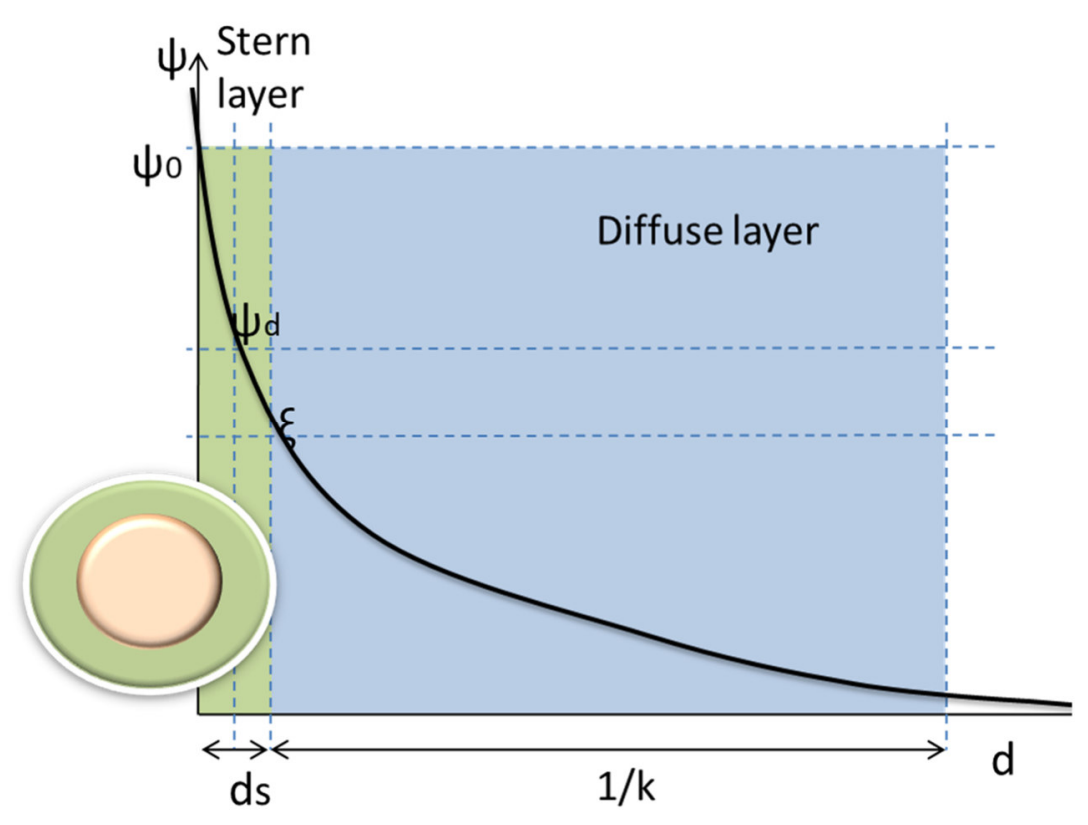

Figure 2 Schematic representation of the structure of the double electrical layer

\section{2. $\quad \mathrm{pH}-$ metry ivestigation}

Using the technique [6], the zero charge point was defined as the intersection point of the curves obtained with increasing the volume of the acid component in the presence of a nanopowder in the suspension composition and with its absence. The results of the study are shown on Figure 3. The data showed that the zero-charge point differs for different types of powders. This point determines the point of the surface potential of the nanoparticle. For the first type of nanoparticles, the zero charge point is $6.8 \mathrm{mV}$, for the second type $-9.6 \mathrm{mV}$. 

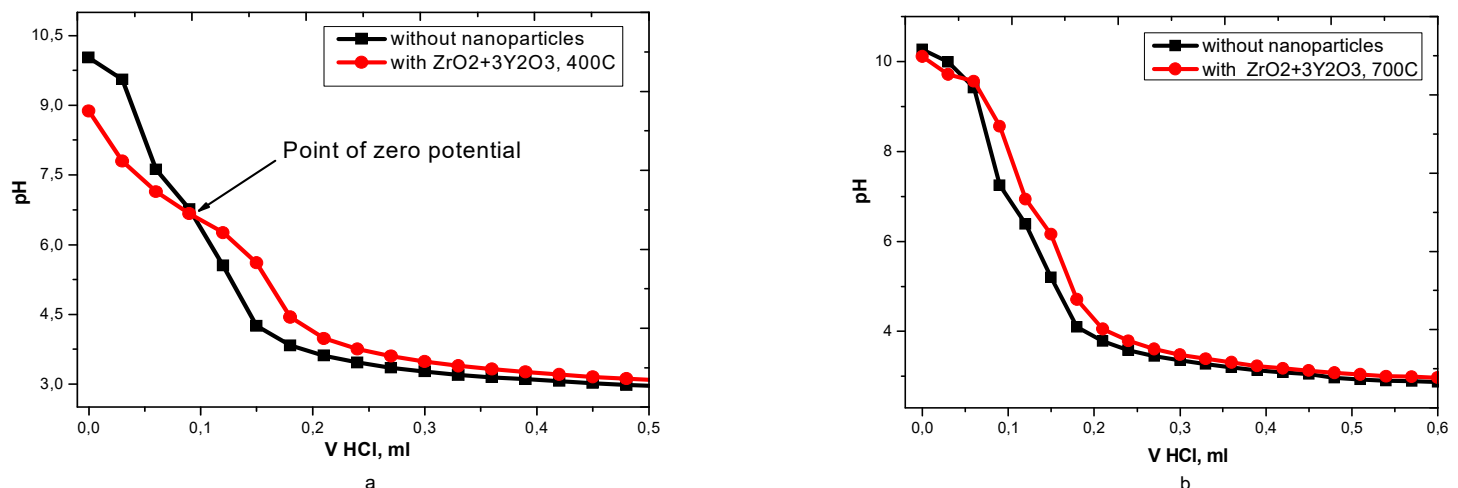

Figure 3 Determination of a point of zero potential for nanopowders with different temperature of calcinations: a) $400{ }^{\circ} \mathrm{C}$; b) $700{ }^{\circ} \mathrm{C}$

The procedure for calculating the basic parameters of a double electric layer is based on these measurements. The main parameters of the double electrical layer are $[11,12,13,14]$ :

Quantity of potential formed ions $\quad \Gamma i=-\frac{C_{H C l}\left(V_{\text {bulk }}-V_{S}\right)}{m \cdot S}$

Total surface charge $\sigma=F \Gamma_{i}$

Surface potential $\varphi_{0}-\psi_{0}=\frac{\sigma}{\varepsilon \varepsilon_{0} \kappa(1+1 / \kappa a)}$

Debye - Huckel Parameter - $\quad \kappa=\left(\frac{2 e^{2} n}{\varepsilon \varepsilon_{0} k T}\right)^{1 / 2}$

Thickness of diffusive layer $d_{d i f f}=1 / \kappa$

Surface charge of Stern layer

$$
\sigma_{1}=\frac{N_{i} z F}{1+\frac{N_{A} v}{M c_{0}} \exp \left(-\frac{z F \psi_{d}+\phi}{R T}\right)}
$$

Surface charge of diffuse layer

$$
\sigma_{2}=\sigma \sqrt{8 c_{0} \varepsilon_{0} K_{d} R T} \sinh \frac{z F \psi_{d}}{2 R T}
$$

Potential of Stern layer

$$
\psi_{\text {Stern }}^{\text {drop }}=\psi_{0}-\psi_{d}=\frac{\sigma_{0} d_{\text {stern }}}{\varepsilon_{0} k_{d}}
$$

Thickness of Stern layer $d_{\text {stern }}=\frac{\varepsilon_{0} k_{d}\left(\psi_{0}-\psi_{d}\right)}{\sigma_{0}}$ 
where $\mathrm{Ni}$ is number of adsorption sites available per square metre of surface to ionic species $\mathrm{i}$ assumed that each site occupied by a water molecyle in a monolayer is a potential cation site, hence $\mathrm{N},=1019 / \mathrm{m}^{2}$, and we found that the results of potential computations are insensitive for $\mathrm{N} ; \mathrm{M}$ is the formula of molar weight of electrolyte $(\mathrm{kg} / \mathrm{mol}) ; \mathrm{v}$ is the density of solvent $\left(\mathrm{kg} / \mathrm{m}^{3}\right) ; \mathrm{c}$ is the molarity (molar concentration) of ions in solution $\left(\mathrm{mol} / \mathrm{m}^{3}\right)$, assuming the electrolyte is symmetrical, i.e., $\mathrm{c},+=\mathrm{c}-; \varphi$ is the specific adsorption potential $(\mathrm{J})$ ( $\varphi=0$ may be assumed for simplified cases); $\varphi d$ is the Stern potential $(V) ; z$ is the valence of cations and anions, assuming electrolyte is symmetrical,' i.e., $z^{+}=z-; F$ is the Faraday constant; $R$ is the gas constant, $T$ is the absolute temperature $(\mathrm{K}) ; \mathrm{Km}$ is the relative permittivity of solvent medium (note the term dielectric constant is used in some literature); and $\varepsilon 0$ is the permittivity of vacuum.

As can be seen from the equations, the values of dielectric permittivity are of great importance in determining the parameters of the double electric layer.

The results of calculations are given in Table 2.

Table 2 The main parameters of the double electroless layer for particles obtained at different annealing temperatures

\begin{tabular}{|l|c|c|}
\hline Parameter & $\mathbf{4 0 0}{ }^{\circ} \mathbf{C}$ & $\mathbf{7 0 0}^{\circ} \mathbf{C}$ \\
\hline $\mathrm{pH}_{\mathrm{ZPC}}$ & 6.8 & 9.6 \\
\hline $\mathrm{pH}_{\text {air-drying }}$ & 5 & 6 \\
\hline$\Gamma_{\mathrm{i}}$ & $1.67^{*} 10^{-7}$ & $1.96^{*} 10^{-6}$ \\
\hline
\end{tabular}

\begin{tabular}{|l|c|c|}
\hline Geometry & $\mathbf{4 0 0}{ }^{\circ} \mathrm{C}$ & $\mathbf{7 0 0}^{\circ} \mathrm{C}$ \\
\hline$D_{\text {stem }}, \mathrm{A}$ & 1.3 & 1.71 \\
\hline $1 / \mathrm{k}, \mathrm{m}^{-1}$ & $3.57^{*} 10^{-8}$ & $7.95^{*} 10^{-8}$ \\
\hline
\end{tabular}

\begin{tabular}{|l|c|c|}
\hline Potential, $\mathbf{V}$ & $\mathbf{4 0 0}{ }^{\circ} \mathbf{C}$ & $\mathbf{7 0 0}{ }^{\circ} \mathbf{C}$ \\
\hline$\phi_{0}$ & 0.420 & 0.468 \\
\hline$\phi_{D}$ & 0.191 & 0.209 \\
\hline$\phi^{\text {drop }}$ & 0.229 & 0.259 \\
\hline Z̈-potential & 0.030 & 0.017 \\
\hline
\end{tabular}

\begin{tabular}{|l|c|c|}
\hline Charge density, $\mathbf{C} / \mathbf{m}^{\mathbf{2}}$ & $\mathbf{4 0 0}{ }^{\circ} \mathbf{C}$ & $\mathbf{7 0 0}^{\circ} \mathrm{C}$ \\
\hline$\sigma_{0}$ & 0.093 & 0.160 \\
\hline$\sigma_{1}$ & 0.0114 & 0.0445 \\
\hline$\sigma_{\text {diff }}$ & 0.082 & 0.116 \\
\hline$\sigma_{\text {兑 }}$ & 0.068 & 0.00356 \\
\hline
\end{tabular}

There are significant changes in the parameters of the double electron layer for different types of nanoparticles. The main differing parameters are the density of the electric charge (twice as high for a nanopowder with a large particle size). A consequence of the different orientation of the water molecules on the surface of the nanoparticle is the different value of the dielectric constant for each of the sublayers of the double electric layer. For particles with a smaller particle size (i.e., when the surface is oriented with oxygen), the values are higher.

According presented data, titration curves of suspensions based on zirconia nanoparticles at different electrolyte concentrations intersect at values other than $\sigma 0=0$, which indicates the occurrence of specific sorption on the surface of zirconia nanoparticles.

\section{CONCLUSIONS}

The acid-base properties of the surface of zirconium dioxide nanoparticles with different particle sizes were studied. It was shown that particles of different sizes have different sorption surface properties, which is manifested in the difference in the main parameters of the double electric layer of nanoparticles. Structure and parameters of double electric layer for different types of zirconia nanoparticles was established. It shows that the value of surface potential at operated $\mathrm{pH}$ levels is higher for particles with larger particle size. 


\section{ACKNOWLEDGEMENTS}

The work was supported through the project H2020/MSCA/RISE/2015/HUNTER GA No69101.

\section{REFERENCES}

[1] PESCHEROVA, N.A., GORBAN, O.A., GORBAN, S.V. Acid-base state of the surface of monoclinic $\mathrm{ZrO}_{2}$ nanoparticles. Scientific works of DonNTU. Series: Chemistry and Chemical Technology. 2010. Vol. 14, no 162, pp. 81-84.

[2] LARSON, lan, ATTARD, Phil. Surface Charge of Silver lodide and Several Metal Oxides. Are All Surfaces Nernstian, J. Colloid Interface Sci. 2000, Vol. 227, pp. 152-163.

[3] DATSKO, T. Ya., ZELENTSOV, V. I. Dependence of the surface charge and fluorine adsorption by Y-aluminum oxide on solution temperature. Electronic processing of materials. 2009. No 5, pp. 65-73.

[4] MAKINO, Kimiko, OSHIMA, Hiroyuki. Electrophoretic Mobility of a Colloidal Particle with Constant Surface Charge Density. Langmuir. 2010. Vol. 26, no 23, pp.18016-18019 https://doi.org/10.1021/la1035745.

[5] KONSTANTINOVA, T. E., DANILENKO, I. A., GLAZUNOVA, V. A., VOLKOVA, G. K., GORBAN, O. A., Mesoscopic phenomena in oxide nanoparticles systems: processes of growth. J. Nanopart. Res. 2011. Vol. 13, p.4015.

[6] PARKS, G. A., de BRYUN, P. L. The zero point of charge of oxides. J. Phys. Chem. 1962. Vol. 66, P. 967.

[7] IKONNIKOVA, K.V., IKONNIKOVA, L.F., MINAKOVA, T.S., SARKISOV, Yu.S. Theory and practice of $p H$-metric determination of acid-base properties of the surface of solids: a training manual. Tomsk: Publishing house of the Tomsk polit. University, 2011, p. 85.

[8] XINMIN, Liu, FEINAN, Hu, WUQUAN, Ding, HANG, Li. A how-to approach for estimation surface/Stern potentials considering ionic size and polarization. The Analyst. 2015, Vol. 140, no 21. https://doi.org/10.1039/c5an01053e

[9] GONGADZE, E., PETERSEN, S., BECK, U., VAN RIENEN, U. Classical Models of the Interface between an Electrode and an Electrolyte. Excerpt from the Proceedings of the COMSOL Conference, 2009, Milan.

[10] BROWN, M.A., GOEL, A., ABBAS, Z. Effect of Electrolyte Concentration on the Stern Layer Thickness at a Charged Interface. Angew Chem Int Ed Engl.2016. Vol.55, no 11, pp. 3790-3794. https://doi.org/10.1002/anie.201512025.

[11] OHSHIMA, Hiroyuki. Potential and Charge of a Hard Particle and a Soft Particle. Chapter 1 Book,First published: 31 January 2012, https://doi.org/10.1002/9781118135440.ch1

[12] OHSHIMA, Hiroyuki. Electrostatic Interaction Between Colloidal Particles. Chapter 2 Book, First published:19 September 2016, https://doi.org/10.1002/9781119075691.ch34

[13] SHANG, J.Q., LO, K.Y., QUIGLEY, R.M. QUANTITATIVE DETERMINATION OF POTENTIAL DISTRIBUTION IN STERN-GOUY DOUBLE-LAYER MODEL. CANADIAN GEOTECHNICAL JOURNAL. 1994. VOL. 31, NO 5, PP.624-636, https://doi.org/10.1139/T94-075.

[14] BROWN, M. A., ABBAS, Z., KLEIBERT, A., GOEL, A., MAY, S., SQUIRES, T.M. Determination of surface potential and electrical double-layer structure at the aqueous electrolyte-nanoparticle interface. Phys.Rev. 2016. Vol. 6, 011007. 